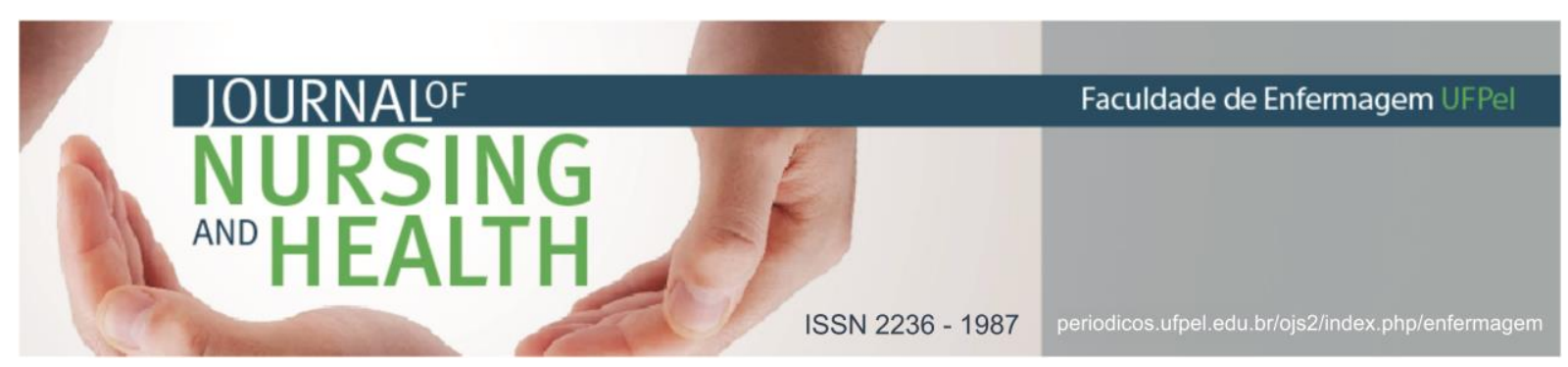

ARTIGO DE REVISÃO

\title{
Prática assistencial dos enfermeiros em atenção primária à saúde: revisão integrativa
}

\author{
Nurse's practice in the primary health care: an integrative review \\ Prácticas asistenciales de los enfermeros en atención primaria a la salud: revisión \\ integradora
}

Ipuchima, Jaqueline Ramires ${ }^{1}$; Souza, Aline Correa²; Weis, Alisia Helena ${ }^{3}$

\begin{abstract}
RESUMO
Objetivo: sintetizar a produção científica brasileira sobre a prática assistencial do enfermeiro em atenção primária à saúde (APS). Métodos: realizou-se uma revisão integrativa no período de janeiro a março de 2016, com busca nas bases de dados LILACS, MEDLINE e SCIELO. Foram selecionados 28 artigos que atenderam aos critérios de inclusão. Resultados: evidenciou-se, que as práticas assistenciais dos enfermeiros ocorrem durante as visitas domiciliares, as consultas de enfermagem e as atividades de vigilância em saúde. Os limites para realização das práticas assistenciais apontados referem-se ao reconhecimento profissional, as relações com a equipe, aos protocolos assistenciais e as capacitações. Considerações finais: destaca-se a inexistência de pesquisas nacionais com evidências fortes relacionados ao tema.

Descritores: Atenção primária à saúde; Enfermagem em saúde comunitária; Cuidados de enfermagem.
\end{abstract}

\section{ABSTRACT}

Objective: to synthesize the Brazilian scientific production on the nursing care practice in the primary health care. Methods: this integrative review was carried out from January to March 2016, by searching on the databases LILACS, MEDLINE and SCIELO. Twenty-eight articles that fitted the inclusion criteria were selected. Results: facilities and limits to the development of health care practices of nurses in the ESF; program activities and evaluation of care practices. Home visits, nursing appointments, and activities surveillance in health accomplish the care practices. The limits to accomplishment indicated in the articles refer to the lack of recognition at work; difficulty in relationship with staff; lack of care protocols and lack of training to perform service activities. Final considerations: there's a lack of national research that stands out strong evidence to the theme. Descriptors: Primary health care; Community health nursing; Nursing care.

\footnotetext{
${ }^{1}$ Enfermeira. Especialista em Saúde Mental. Enfermeira da Irmandade Santa Casa de Misericórdia de Porto Alegre, Porto Alegre, Rio Grande do Sul, Brasil. Email: jaqueramires@hotmail.com http://orcid.org/0000-0002-74460566

2 Enfermeira. Doutora em Enfermagem. Docente da Universidade Federal de Ciências da Saúde de Porto Alegre , Porto Alegre, Rio Grande do Sul, Brasil. Email: alinec@ufcspa.edu.br http://orcid.org/0000-0001-5824-3468

${ }^{3}$ Enfermeira. Doutora em Enfermagem. Docente da Universidade Federal de Ciências da Saúde de Porto Alegre, Porto Alegre, Rio Grande do Sul, Brasil. Email: alisia@ufcspa.edu.br http://orcid.org/0000-0003-4830-4583
} 


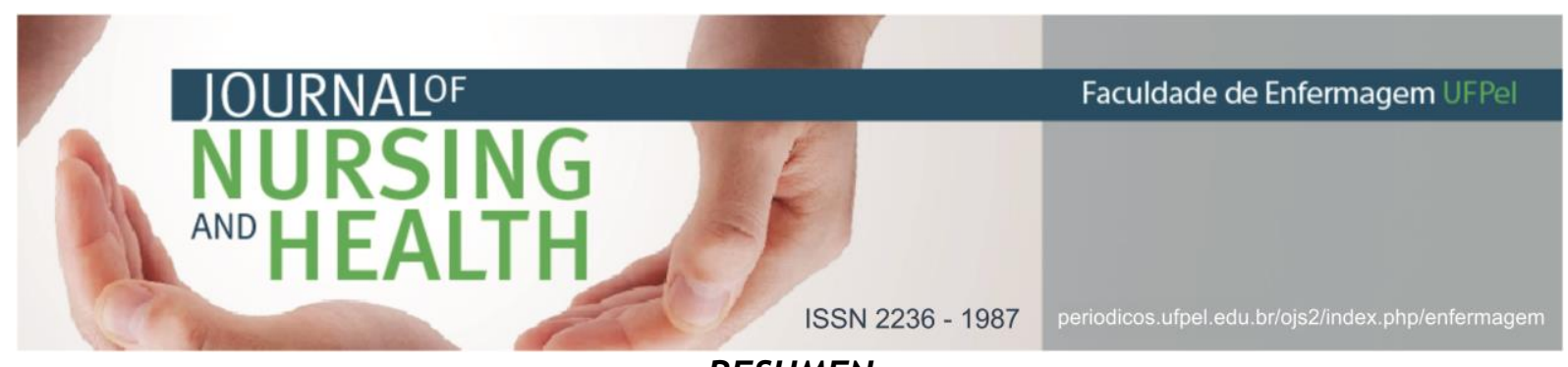

RESUMEN

Objetivo: resumir la literatura científica nacional sobre el bienestar de las enfermeras en la atención primaria de salud (APS). Métodos: una revisión integradora que se llevó a cabo en el período eneromarzo de 2016, con búsqueda en las bases de datos de LILACS, MEDLINE y SCIELO. Fueron seleccionados 28 artículos que cumplían con los criterios de inclusión. Resultados: se evidenció que las prácticas asistenciales de los enfermeros se pasan durante las visitas a los hogares, las consultas de enfermería y las actividades de vigilancia en salud. Los límites para la realización de las prácticas asistenciales fijados hablan del reconocimiento profesional, las relaciones con el equipo, a los protocolos asistenciales y las capacitaciones. Consideraciones finales: se destaca la ausencia de investigaciones nacionales con evidencias fuertes relacionadas al tema.

Descriptores: Atención primaria de salud; Enfermería en salud comunitaria; Atención de enfermería.

\section{INTRODUÇÃO}

A Atenção Primária a Saúde (APS), corresponde ao nível de atenção que oferece entrada ao sistema de saúde para necessidades e problemas de saúde das pessoas, pode ser entendida como uma abordagem que forma a base e determina o trabalho de todos os outros níveis do sistema. ${ }^{1}$ Tem como por característica a prestação de ações em conjunto, tanto individuais como coletivas, com complexidade elevada e densidade baixa. Ela compreende a realização de práticas de promoção e proteção da saúde, bem como a prevenção de agravos, diagnóstico, tratamento e a reabilitação. ${ }^{2}$

Neste nível de atenção os enfermeiros desenvolvem práticas assistenciais, gerenciais e educativas. ${ }^{3-}$ 6 Essas práticas estão voltadas para indivíduos e famílias em unidades de saúde, nos diferentes ciclos vitais, em domicílio e nos espaços comunitários, exercendo, predominantemente, consultas de enfermagem, visitas domiciliares e grupos educativos e atividades com a equipe de saúde. ${ }^{3}$

Estas práticas estão regulamentadas pela Lei do Exercício Profissional da Enfermagem - LEP 7.498/86 que normatiza o desenvolvimento das atividades dos enfermeiros nos serviços de saúde. Desse modo, as atividades desenvolvidas pelos enfermeiros são práticas sociais que vão além da dimensão técnica e profissional, incluem a produção do conhecimento, a reprodução socioeconômica e política e a inserção dos sujeitos nesse processo. $^{7}$

$\mathrm{Na}$ especificidade das práticas assistenciais dos enfermeiros, entende-se que são ferramentas para a consolidação da APS abrangente, visto que conseguem organizar serviços pautados na produção do cuidado, modelando o trabalho em saúde. ${ }^{8}$ As práticas assistenciais ampliam a meta do cuidado, pois produzem atividades clínicas direcionadas para os diferentes níveis de idade, bem como acolhem à demanda programa, espontânea, do território e da comunidade. ${ }^{9}$

No contexto da APS os enfermeiros têm assumido papel estratégico na constituição do sistema de cuidado. Além de agregarem diversos saberes, possuem também a capacidade de interação com os demais profissionais da equipe, permitindo assim uma autonomia para 
avaliação das necessidades dos usuários, norteando sua prática assistencial. ${ }^{10}$ Tais atividades contemplam ações fundamentadas no saber clínico e epidemiológico, realizadas de forma articulada no cotidiano das práticas.

Assim sendo, a APS é um campo de atuação complexo e promissor para o enfermeiro, à medida que conseguem, dentro dessa área, desempenhar seu trabalho com autonomia, bem como atingindo reconhecimento social. ${ }^{11}$

Diante do caráter inovador da APS, que têm na Estratégia de Saúde da Família (ESF) sua principal ferramenta para reorganizar a $A B S$, torna-se importante conhecer de que forma a produção científica brasileira sobre a prática assistencial do enfermeiro, tem contribuído para a visibilidade da clínica e para a consolidação do modelo de atenção no SUS. Diante do contexto apresentado, questiona-se: qual é a produção científica brasileira sobre a prática assistencial do enfermeiro na APS?

Assim, com o propósito de ampliar as discussões acerca da atuação do enfermeiro na APS, foi realizado este estudo com objetivo de sintetizar a produção científica brasileira sobre a prática assistencial do enfermeiro na APS.

\section{MATERIAIS E MÉTODOS}

Realizou-se uma revisão integrativa. Este método trata da análise de pesquisas que possuem relevância e que dão suporte para tomadas de decisões, com a finalidade de melhorar a prática clínica, permitindo ainda que ocorra uma síntese dos diversos estudos publicados, possibilitando conclusões referentes a respeito de uma área de estudo. ${ }^{12}$

As etapas seguidas para a organização da revisão foram: identificação do tema e seleção da hipótese ou questão de pesquisa; estabelecimento de critérios para inclusão e exclusão; categorização dos estudos; avaliação dos estudos incluídos; interpretação dos resultados e síntese do conhecimento. ${ }^{12}$

A questão norteadora que orientou a identificação do problema foi: Qual a produção de conhecimento sobre a prática assistencial do enfermeiro em atenção primária à saúde no Brasil?

Foram considerados critérios de inclusão os artigos brasileiros resultado de pesquisa, publicados até 2005 2015 nos idiomas português, inglês e espanhol com acesso online e gratuito do texto completo. Foram incluídos artigos que abordassem o tema trabalho em equipe que destacassem em categoria específica as práticas dos enfermeiros. Os critérios de exclusão foram: teses, dissertações, publicações duplicadas, estudos de revisão, reflexão e relatos de experiência e os que não respondiam à questão de pesquisa.

Os Descritores em Ciências da Saúde (DeCs/MESH) utilizados foram: "Atenção Primária à Saúde", "Enfermagem de Atenção Primária", "Papel do Profissional de Enfermagem", "Prática Clínica Baseada em Evidências", "Prática de Saúde Pública”, "Programa de Saúde 


\section{JOURNALOF \\ NURSING \\ ANO HEALTH}

ISSN 2236 - 1987

espanhol não apresentarem retorno nas buscas, referente a produção brasileira.

Após a leitura do título e dos resumos, foram identificadas 73 publicações em potencial, referente a prática assistencial dos enfermeiros em atenção primária à saúde. Posteriormente foi realizada a leitura na íntegra dos estudos. Dos 73 artigos, foram excluídos 45 artigos, por não responderem à questão de pesquisa ou estarem repetidos nas bases de dados. Dessa forma, a amostra final foi constituída por 28 artigos.

As buscas nas bases de dados, a seleção dos artigos e a classificação das evidências foram realizadas por dois avaliadores e na discordância de algum estudo excluído, um terceiro avaliador foi consultado.

Foi elaborado um roteiro para a avaliação dos artigos. O roteiro foi composto das seguintes informações: título, autor(es), revista, combinação dos descritores utilizados, bases de dados, objetivo, metodologia, nível de evidência, periódico e ano.

Para a classificação dos artigos por nível de evidência, foi empregado o sistema de classificação do nível de evidência de Melnyk e FineoutOverholt $^{13}$ (2005). Este sistema é composto por sete níveis, sendo: Nível I - evidências originárias de revisões sistemáticas ou meta-análise de relevantes ensaios clínicos; Nível II evidências derivadas de pelo menos um ensaio clínico randomizado controlado bem delineado; Nível III - ensaios clínicos bem delineados sem randomização; Nível IV - estudos de coorte e de caso-controle bem 


\section{NURSING \\ AND HEA}

delineados; Nível $\mathrm{V}$

revisão sistemática de estudos descritivos e qualitativos; Nível VI - evidências derivadas de um único estudo descritivo ou qualitativo e Nível VII opinião de autoridades ou relatório de comitês de especialistas.

A análise de dados ocorreu em duas etapas. $\mathrm{Na}$ primeira etapa foi realizada a caracterização do perfil das publicações através das informações extraídas do roteiro de coleta de dados. Foram utilizados cálculos de frequência simples e relativa. $\mathrm{Na}$ segunda etapa procedeu-se a leitura detalhada das publicações e se realizou a análise do conteúdo dos artigos. Os dados foram tratados pela técnica de análise de conteúdo temática. ${ }^{14}$

Nesta revisão integrativa é assegurada a autoria dos artigos pesquisados, de forma que todos os estudos utilizados são referenciados. Por não envolver seres humanos, não foi necessária a aprovação do projeto de pesquisa em Comitê de Ética em Pesquisa.

\section{RESULTADOS E DISCUSSÃO}

A amostra final constituiu-se de 28 artigos. 0 período de maior publicação foi de 2010 a 2013 com 23 artigos $(82,1 \%)$. 0 ano de maior produção foi 2011 com nove artigos (32,1\%) publicados e seguido pelos anos de 2012 com seis $(21,4 \%), 2013$ e 2010 com quatro $(14,3 \%)$ artigos cada. Em 2005, 2006 e 2008 foram publicados apenas um artigo em cada ano (3,5\%) seguido de dois artigos em 2008.

Os periódicos que mais publicaram trabalhos sobre o tema
ISSN 2236 - 1987

foram a Revista de Enfermagem UERJ e a Escola Anna Nery Revista de Enfermagem, sendo publicados cinco artigos (17,8\%), a seguir a Revista Acta Paulista de Enfermagem, a Revista Texto e Contexto Enfermagem e a Revista da Escola da Enfermagem da USP com três artigos (10,7\%) cada uma delas. $\mathrm{Na}$ Revista Gaúcha de Enfermagem, dois artigos $(7,1 \%)$ publicados no período. Os periódicos Interface - Comunicação, Saúde Educação, Jornal Brasileiro de Psiquiatria, Online Brazilian Journal of Nursing, Revista Brasileira de Enfermagem, Revista Ciência \& Saúde Coletiva, Revista Latino-Americana de Enfermagem e Revista Psicologia em Estudo foram os que tiveram o menor número de publicações no período, ou seja um artigo $(3,6 \%)$ em cada uma delas nos anos estudados.

Os métodos de pesquisa mais utilizados nos artigos encontrados foram: qualitativa, 15 artigos (53,6\%); quantitativa, nove artigos (32,1\%); quanti-qualitativo e teóricos dois artigos cada um (7,1\%). Analisando-se o nível de evidência, $100 \%$ dos artigos tiveram nível de evidência classificado como fracas (níveis de $\mathrm{V}$ a VII). A metodologia descritiva com análise qualitativa foi a mais utilizada.

Quanto ao conteúdo dos artigos, foi evidenciado duas temáticas no estudo: atividades assistenciais desenvolvidas pelos enfermeiros e facilidades e limites para 0 desenvolvimento da assistência.

\section{Atividades assistenciais desenvolvidas pelos enfermeiros}

Foram encontrados 19 artigos que apontaram as práticas assistenciais dos 
enfermeiros, que estão vinculadas as ações programáticas e aos programas do Ministério da Saúde. As ações apontadas nos estudos englobam a consulta de enfermagem e a visita domiciliar ${ }^{15-16}$ vigilância em saúde (saúde ambiental e vigilância epidemiológica) ${ }^{9,17-19}$; saúde do homem. ${ }^{20}$

Dentro as consultas de enfermagem encontraram-se as atividades voltadas para a saúde da mulher, puericultura, hipertensão, diabetes e acolhimento ${ }^{21}$; orientações em saúde sobre malefícios e benefícios do uso e abuso de álcool e drogas, risco para a gestação e desenvolvimento de doenças crônicas ${ }^{22}$; acolhimento, agendamento de consultas, encaminhamento para pediatra e teste do pezinho ${ }^{23}$; atividades educativas para adolescentes, envolvendo o planejamento familiar, pré-natal, exame preventivo de câncer uterino, orientação sobre uso de preservativo, gravidez e DST/AIDS. ${ }^{24}$

$\mathrm{Na}$ visita domiciliar, o enfermeiro identifica necessidades de saúde, planeja, mobiliza a equipe, executa, acompanha e a avalia as ações de saúde. ${ }^{25}$ Além de promover 0 acolhimento das demandas dos usuários, destacam-se os atendimentos individuais dos portadores de doenças crônicas, bem como a investigação das necessidades da saúde da família, prática utilizada como instrumento para o cuidado em saúde e organização do trabalho da equipe da ESF. A pesquisa traz o papel do enfermeiro associado à investigação, orientação, intervenção de enfermagem, como curativo, vacinação, coleta de exames,
ISSN 2236 - 1987

verificação de hemoglicoteste. ${ }^{15}$

A visita domiciliar funciona como um importante instrumento para a prestação da assistência à saúde do indivíduo, uma vez que traz resultados inovadores, pois há possibilidade de conhecer a realidade do paciente e de sua família in loco, além do fortalecimento de vínculos. ${ }^{26}$ As visitas domiciliares, além do cunho técnico, como foi apresentado acima, devem prover atividades que permitam a criação de vínculos com a comunidade e seus usuários bem como, atuar no desenvolvimento da humanização do atendimento da população. ${ }^{27}$

As práticas voltadas para a vigilância em saúde focalizam a saúde ambiental, que é realizada através do reconhecimento das situações ambientais, que impliquem em risco potencial à saúde humana e da comunidade ${ }^{17} \mathrm{e}$ da prevenção de agravos à saúde no cotidiano de trabalho. ${ }^{18}$ Incorporando as práticas de saúde ambiental ao seu cotidiano, os enfermeiros tendem a ampliar seu objeto de trabalho, o cuidado, consequentemente, amplia-se o campo de atuação que passa a compreender melhor a relação do homem com o ambiente e assim consegue exercer a promoção de saúde. ${ }^{28}$

As práticas voltadas para 0 controle da tuberculose ${ }^{9}$ envolvem a notificação dos casos, convocação dos doentes, orientação para coleta de escarro e busca ativa dos faltosos.

A atuação do enfermeiro, também foi abordada na perspectiva da hanseníase, onde o profissional realiza o planejamento e execução das 


\section{JOURNALOF \\ NURSING \\ AND HEALTH}

ISSN 2236 - 198

como um meio de prática assistencial do enfermeiro em ESF.

Sobre a saúde ambiental, os enfermeiros também têm sua prática assistencial através da vigilância, dizem que incorporando as práticas de saúde ambiental ao seu cotidiano de trabalho os enfermeiros tendem a ampliar seu objeto de trabalho, o cuidado. ${ }^{28}$

É incontestável a relação entre saúde e meio ambiente, portando essa abordagem ambiental, na construção do trabalho do enfermeiro é importante para o controle dos agravos. Porém ainda existem lacunas na abordagem da relação saúdeambiente, mostrando que a discussão sobre a relação saúde e meio ambiente é um desafio na formação profissional em enfermagem. ${ }^{30}$

\section{Facilidades e limites para o desenvolvimento da assistência}

Quanto às facilidades e limites para o desenvolvimento das práticas assistenciais dos enfermeiros em APS, nove artigos foram encontrados.

As facilidades destacam-se a Sistematização da Assistência de Enfermagem (SAE) como importante instrumento para a garantia da qualidade assistencial. ${ }^{31} 0$ cuidado prolongado com a população estabelece vínculos com boa duração. ${ }^{32} 0$ reconhecimento e valorização do enfermeiro pela comunidade, a realização de capacitações para o cuidado e educação permanente. ${ }^{33}$

0 trabalho do enfermeiro em ESF pode trazer uma série de desafios a serem superados, gerando inclusive 


\section{JOURNAIOF

carga de trabalho extra, contudo, o trabalho dos profissionais enfermeiros, gera ampla participação destes na estruturação de melhores práticas de saúde. ${ }^{34}$

Os limites para as atividades assistenciais dos enfermeiros na APS, identificados nos artigos estão relacionados a clínica voltada para o modelo curativista, educação em serviços e infraestrutura insuficientes.

As práticas assistenciais do enfermeiro na saúde do idoso esbarram na ineficiência do cuidado, por falta de capacitações e de recursos materiais, resumindo-se às visitas domiciliares e cuidados a hipertensos e diabéticos através de determinações sobre os hábitos de vida. ${ }^{35}$

Já as ações dos enfermeiros voltadas para usuários de álcool e drogas, estão direcionadas na perspectiva tradicional de atenção à saúde. As ações estão voltadas para o atendimento de comorbidades, através da solicitação de exames laboratoriais, agendamento para consulta de enfermagem, realização de curativos e feridas, aferição de pressão arterial e acompanhamento ao portador de HIV. ${ }^{36}$ A abordagem aos usuários de álcool e drogas na APS é um dos desafios das equipes de saúde.

Sobre a Atenção Integrada às Doenças Prevalentes na Infância (AIDPI), os limites encontrados estão relacionados a carência de conhecimento dos profissionais sobre este modelo e o protocolo nos serviços de saúde, além da falta de insumos e inadequações na estrutura física das unidades de saúde para o atendimento a criança. ${ }^{37}$
Sobre a práxis da consulta de enfermagem para o cuidado infantil, também é apontado problemas nas questões estruturais do ambiente de trabalho, como precariedade física, as quais influenciam diretamente na qualidade da consulta de enfermagem. ${ }^{38} \quad$ Estudos $^{39-40}$ corroboram com estes achados, apontando que a estrutura física e tecnológica inadequada e a falta de recursos materiais são entraves para a prática do enfermeiro, pois repercutem na qualidade e na eficiência da assistência prestada ao cliente.

Problemas relacionados às atividades de educação em serviços e a insuficiência na realização de ações de capacitação para a realização das práticas assistenciais direcionadas para a saúde mental, para as gestantes e para os adolescentes são apontados em alguns estudos. ${ }^{41-43}$ As práticas dos enfermeiros a esses grupos estão pautadas em consultas de enfermagem e visita domiciliar, com foco nas questões tradicionais e rotineiras. ${ }^{41-43}$

Pensando no aperfeiçoamento e no fortalecimento da APS, bem como da consolidação dos princípios básicos do SUS, há necessidade de que os enfermeiros sejam capazes de reconhecer e entender o contexto dos problemas sociais da comunidade, que possam junto com a equipe e com os gestores da saúde, criar meios de intervir nas atividades assistenciais e na organização do setor da saúde. ${ }^{43}$

\section{CONSIDERAÇÕES FINAIS}

Os estudos analisados nesta revisão permitiram identificar e sintetizar as evidências disponíveis na 


\section{JOURNALOF \\ NURSING \\ AND HEALTH}

ISSN 2236 - 1987

gestores, também foi reconhecida como um limitador para as práticas assistências qualificadas. Todos os estudos apontaram evidências fracas nessa área de conhecimento, com base nos níveis de evidência $V$ a VII. Além disso, há falta de especificidade nos artigos sobre a clínica do enfermeiro nas práticas assistenciais. Não há publicações brasileiras em revistas internacionais.

Contudo, indica-se a realização de novos estudos no cenário nacional com evidências fortes, através de ensaios clínicos e revisões sistemáticas, que explorem a clínica do enfermeiro no sentido de se avançar o conhecimento sobre a prática assistencial do enfermeiro na APS e de visibilizar o cuidado realizado em produções científicas.

\section{REFERÊNCIAS}

1. Starfiled, B. Atenção primária: equilíbrio entre necessidades de saúde, serviços e tecnologias. $1^{\mathrm{a}}$ ed. Brasília: UNESCO: Ministério da Saúde; 2002.

2. Ministério da Saúde (BR). Secretaria de Atenção Básica. PNAB - Política Nacional de Atenção Básica. Brasília; 2006.

3. Colomé ICS. Prática clínica das enfermeiras na estratégia saúde da família: exercendo a clínica do cuidado [tese]. Porto Alegre (RS): Escola de Enfermagem da Universidade Federal do Rio Grande do Sul; 2013.

4. Kantorski LP, Jardim VMR, Coimbra VCC, Oliveira MM, Heck RM. A integralidade da atenção à saúde na perspectiva da gestão no município. 


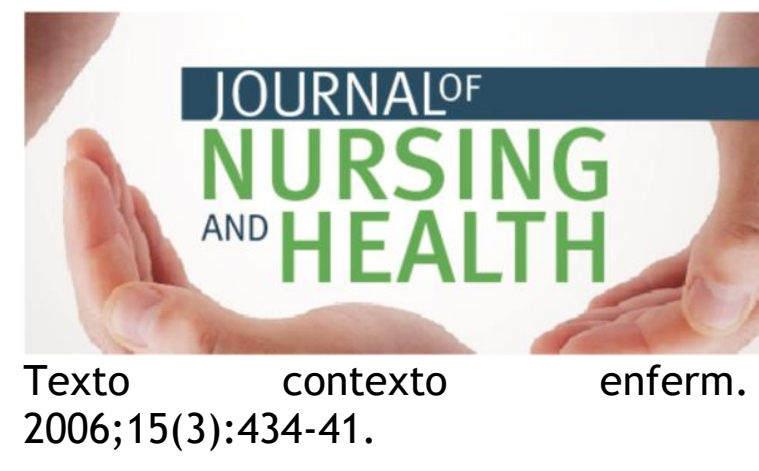

5. Weirich CF, Munari DB, Mishima SM, Bezerra ALQ. O trabalho gerencial do enfermeiro na Rede Básica de Saúde. Texto contexto enferm. 2009;18(2):249-57.

6. Matumoto $S$, Fortuna $C M$, Kawata LS, Mishima SM, Pereira MJB. A prática clínica do enfermeiro na atenção básica: um processo em construção. Rev latino-am enfermagem. 2011;19(1):123-30.

7. Nauderer TM, Lima MADS. Práticas de enfermeiros em unidades básicas de saúde em município do sul do Brasil. Rev latino-am enfermagem. 2008;16(5):889-94.

8. Conill EM, Fausto MCR, Giovanella L. Contribuições da análise comparada para um marco abrangente na avaliação de sistemas orientados pela atenção primária na América Latina. Rev bras saúde matern infant. 2010;10(1):15-27.

9. Santos AM, Giovanella L, Mendonça MHM, Andrade CLT, Martins MIC, Cunha MS. Práticas assistenciais das equipes de saúde da família em quatro grandes centros urbanos. Cienc saúde colet. 2012;17(10):2687-702.

10. Nascimento KC, Backes DS, Koerich MS, Erdmann AL. Sistematização da assistência de enfermagem: vislumbrando um cuidado interativo, complementar e multiprofissional. Rev esc enferm USP. 2008;42(4):643-8.

11. Costa RKS, Miranda FAN. O enfermeiro e a Estratégia Saúde da Família: contribuição para a mudança do modelo assistencial. Rev RENE. 2008;9(2):120-8.
ISSN 2236 - 1987

12. Mendes KDS, Silveira RCCP, Galvão CM. Revisão integrativa: método de pesquisa para a incorporação de evidências na saúde e na enfermagem. Texto contexto enferm. 2008;7(4):75864.

13. Melnik BM, Fineout-Overholt E. Making the case for evidence-based practice and cultivating a spirit of inquiry. In: Melnyk BM, FineoutOverholt $\mathrm{E}$. Evidence-based practice in nursing \& healthcare. A guide to best practice. Philadelphia, PA: Lippincott Williams \& Wilkins; 2005.

14. Minayo, MCS. O desafio do conhecimento: pesquisa qualitativa em saúde. $12^{\mathrm{a}}$ ed. São Paulo: Hucitec; 2010.

15. Kebian LVA, Acioli S. Visita domiciliar: espaço de práticas de cuidado do enfermeiro e do agente comunitário de saúde. Rev enferm UERJ. 2011;19(3):403-9.

16. Lionello CDL, Duro CLM, Silva AM, Witt RR. O fazer das enfermeiras da estratégia de saúde da família na atenção domiciliária. Rev gauch enferm. 2012;33(4):103-10.

17. Cezar-Vaz MR, Weis AH, Costa VZ, Soares JFS, Bonow CA, Cardoso LS, et al. Estudo com enfermeiros e médicos da atenção básica à Saúde: uma abordagem socioambiental. Texto contexto enferm. 2007:16(4):645-53.

18. Pelegrini AHW, Cezar-Vaz MR. Atenção primária ambiental no trabalho das enfermeiras da rede básica de saúde: um estudo de corte transversal. Online braz j nurs [Internet]. 2010[acesso em 2016 ago 10];9(1). Disponível em: http://www.objnursing.uff.br/index. 


\section{JOURNALOF \\ NURSING \\ AND HEALTH}

php/nursing/article/view/j.1676-

4285.2010.2851/638

19. Lanza FM, Lana FCF. O processo de trabalho em hanseníase: tecnologias e atuação da equipe de saúde da família. Texto contexto enferm. 2011;20(esp):238-46.

20. Silva PAS, Furtado MS, Guilhon $A B$, Souza NVDO, David HMSL. A saúde do homem na visão dos enfermeiros de uma unidade básica de saúde. Esc Anna Nery. 2012:6(3):561-8.

21. Ermel RC, Fracolli LA. O trabalho das enfermeiras no Programa de Saúde da Família em Marilia/SP. Rev esc enferm USP. 2006;40(4):533-9.

22. Souza ICW, Ronzani TM. Álcool e drogas na Atenção Primária: avaliando estratégias de capacitação. Psicol estud. 2012;17(2):237-46.

23. Souza MHN, Paz EPA, Farias SNP, Ghelman LG, Mattos CX, Barros RR. Integralidade como uma dimensão da prática assistencial do enfermeiro no acolhimento mãe-bebê. Esc Anna Nery. 2013;17(4):677-82.

24. Ferrari RAP, Thomson Z, Melchior R. Adolescência: ações e percepção dos médicos e enfermeiros do Programa Saúde da Família. Interface (Botucatu). 2008;12(25):387-400.

25. Fortuna CM, Matumoto S, Pareira MJB, Mishima SM, Kawata LS, CamargoBorges C. $O$ enfermeiro e as práticas de cuidados coletivos na estratégia saúde da família. Rev latino-am enferm. 2011;19(3):8 telas.

26. Mourão SM, Freitas CASL, Dias MAS, Lobo MGP. A visita domiciliar como instrumento para a promoção de práticas de higiene: uma revisão bibliográfica. Sanare. 2010;9(2):86-92.
ISSN 2236 - 198

27. Noro LRA, Torquato SM. Visita domiciliar: estratégia de aproximação à realidade social? Trab educ saúde. 2015;13(1):145-58.

28. Lopes MSV, Ximenes LB. Enfermagem e saúde ambiental: possibilidades de atuação para a promoção da saúde. Rev bras enferm. 2011;64(1):72-7.

29. Leão LHC, Vasconcellos LCF. Cadeias produtivas e a vigilância em saúde, trabalho e ambiente. Saude soc. $2015 ; 24(4): 1232-43$.

30. Peres RR, Camponogara S, Costa VZ, Terra MG, Nietsche EA. Saúde e ambiente: (in) visibilidades e (des) continuidade na formação profissional em enfermagem. Esc Anna Nery. 2016;20(1):25-32.

31. Souza RS, Siqueira MM. O processo de enfermagem na assistência a pacientes com dependência de álcool. J bras psiquiatr. 2005;54(3): 228-33.

32. Baratieri T, Marcon SS. Longitudinalidade no trabalho do enfermeiro: identificando dificuldades e perspectivas de transformação. Texto contexto enferm. 2012;21(3):549-57.

33. Beck CLC, Prochnow A, Silva RM, Prestes FC, Tavares JP. Fatores que favorecem e dificultam o trabalho dos enfermeiros nos serviços de atenção à saúde. Esc Anna Nery. 2010;14(3):4905.

34. Melo RC, Machado ME. Coordenação de unidades de saúde da família por enfermeiros: desafios e potencialidades. Rev gauch enferm. 2013;34(4):61-7.

35. Rocha FCV, Carvalho CMRG, Figueiredo MLF, Caldas CP. O cuidado 


\section{NURSING \\ AND HEALTH}

do enfermeiro ao idoso na Estratégia de Saúde da Família. Rev enferm UERJ. 2011;19 (2):186-91.

36. Gonçalves SSPM, Tavares CMM. Atuação do enfermeiro na atenção ao usuário de álcool e outras drogas nos serviços extra - hospitalares. Esc Anna Nery. 2007;11(4):586-92.

37. Higuchi $\mathrm{CH}$, Fujimori E, Cursino EG, Chiesa AM, Veríssimo MDLOR, Mello DF. Atenção Integrada às doenças prevalentes na infância (AIDPI) na prática de enfermeiros egressos da USP. Rev gaúch enferm. 2011;22(2):241-7.

38. Waidman MAP, Marcon SS, Pandini A, Bessa JB, Paiano M. Assistência de enfermagem às pessoas com transtornos mentais e às famílias na Atenção Básica. Acta paul enferm. 2012;25(3):346-51.

39. Rodrigues EM, Nascimento RG, Araujo A. Protocolo na assistência prénatal: ações, facilidades e dificuldades dos enfermeiros da Estratégia de Saúde da Família. Rev esc enferm USP. 2011;45(5):1041-7.

40. Higarashi IH, Baratieri T, Roecker S, Marcon SS. Atuação do enfermeiro junto aos adolescentes: Identificando dificultades e perspectivas de transformação. Rev enferm UERJ. 2011;19(3):375-80.

41. Saparolli ECL, Adami NP. Avaliação da estrutura destinada à consulta de enfermagem à criança na atenção básica. Rev esc enferm USP. 2010;44(1):92-8.

42. Roecker S, Budó MLD, Marcon SS. Trabalho educativo do enfermeiro na Estratégia Saúde da Família: dificuldades e perspectivas de
ISSN 2236 - 1987

mudanças. Rev esc enferm USP. 2012;46(3):641-9.

43. Xavier-Gomes LM, Andrade-Barbosa TL, Silva CSO, Lopes JR, Leite MTS. Prática gerencial do enfermeiro na estratégia de saúde da família. Trab educ saúde. 2015;13(3):695-707.

Data de submissão: 13/04/2017

Data de aceite: 09/11/2017

Data de publicação: 26/12/2017 